\title{
A CHARACTERIZATION OF UNCONDITIONED WEAK SEQUENCES
}

\author{
MANFRED STEURICH'
}

\begin{abstract}
Unconditioned weak sequences are characterized in terms of the Koszulhomology and applications to the theory of Buchsbaum rings and in a special case to the resolution of the ideal generated by the weak sequence are considered
\end{abstract}

Introduction. In the theory of local rings there has been a rapid development of the theory of Buchsbaum rings, for basic facts see $[8,4,5,6]$. Buchsbaum rings may be defined by the property that for every system of parameters $x$ of the local ring $(R, \mathfrak{m})$ the difference of length $(R /(\mathfrak{x}))$ and multiplicity of the ideal $(\mathfrak{x})$ is an invariant, i.e. does not depend on the choice of the system of parameters $x$, or by the property: every system of parameters $x_{1}, \ldots, x_{n}$ is a weak sequence, i.e.

$$
\mathrm{m}\left(\left(x_{1}, \ldots, x_{i-1}\right): x_{i}\right) \subseteq\left(x_{1}, \ldots, x_{i-1}\right) \text { for all } 1 \leqslant i \leqslant n .
$$

In the following we will say that a sequence of elements $x_{1}, \ldots, x_{n}$ is an unconditioned weak sequence if it satisfies (*) for any renumbering. Hence every system of parameters in a Buchsbaum ring is certainly an unconditioned weak sequence.

Another characterization of Buchsbaum rings follows from the result in [7], saying: for every system of parameters $\mathfrak{x}$ the Koszulhomology $H_{*}(\mathfrak{x})$ is a vectorspace, equivalently $H_{1}(\mathfrak{x})$ is a vectorspace. Hence it is equivalent demanding the weak sequence property for all systems of parameters $\mathfrak{x}$ of a local ring and demanding $H_{*}(\mathfrak{x})\left(H_{1}(\mathfrak{x})\right)$ is a vectorspace for all such $\mathfrak{x}$. So one may ask: given any sequence $x_{1}, \ldots, x_{n}$ in a local ring, what does it mean for the Koszulhomology if we demand $x$ to be an unconditioned weak sequence?

In this paper we want to answer this question. It turns out that the assumption for $x$ to be an unconditioned weak sequence does not imply in general that the Koszulhomology modules $H_{i}(\mathfrak{x})$ for $i \geqslant 1$ are vectorspaces, but that $H_{i}(\mathfrak{x})$ modulo a certain submodule is a vectorspace. These submodules vanish e.g. if $r$ has the additional property to be unconditioned relatively regular in the sense of Fiorentini [1]. For a system of parameters $x$ of a Buchsbaum ring this property of course holds, since $x$ is even a (unconditioned) $d$-sequence (see [2]).

Further we show that for an unconditioned weak sequence $x$ the Koszulcomplex is always a (module-)direct summand of the minimal $R$-free resolution of $R /(\mathfrak{x})$ for $n \leqslant 2$. We then show that if for every multi-index $\left(\nu_{1}, \ldots, \nu_{n}\right)$ the syzygetic part of

Received by the editors July 16, 1981.

1980 Mathematics Subject Classification. Primary 13 H99.

'Supported by the DFG (Deutsche Forschungsgemeinschaft). 
the Koszulhomology $\tilde{H}_{1}\left(x_{1}^{\nu_{1}}, \ldots, x_{n}^{\nu_{n}} ; M\right)$ is a vectorspace for a finitely generated $R$-module $M$, then $x_{1}^{\nu_{1}}, \ldots, x_{n}^{\nu_{n}}$ is a weak sequence on $M$ for all $\left(\nu_{1}, \ldots, \nu_{n}\right)$. Hence from this fact and our characterization of weak sequences the characterization of Buchsbaum rings derived from Suzuki [7] follows and, more generally, together with the result in [7], this gives another proof of Shimoda's characterization of Buchsbaum modules (cf. [3]).

Finally we consider, in the case that the $H_{i}(x)$ are vectorspaces, the resolution of $R /\left(x_{1}, x_{2}\right)$ as an $R$-module for an unconditioned weak sequence $x_{1}, x_{2}$ and give a condition when this resolution is obtained by the Koszulcomplex and copies of the resolutions of the residue class field suitably lifted.

The author would like to thank S. Goto for the stimulating discussions at Brandeis University.

Notations. All rings $(R, \mathrm{~m})$ will be commutative with 1 , noetherian and local with maximal ideal $m$. Let $x_{1}, \ldots, x_{n}$ be elements of $R$. We will write $\mathfrak{x}$ for the whole sequence $x_{1}, \ldots, x_{n} ;(\mathfrak{x})$ denotes the $R$-ideal generated by $\mathfrak{x}$. For any subset $I \subseteq$ $\{1, \ldots, n\}$ and any $i \in I$ we write $I_{i}$ for the subset of all elements of $I$ excluding $i$. Also $x_{1}, \ldots, \hat{x}_{i}, \ldots, x_{n}$ stands for the sequence with $x_{i}$ omitted.

Let $I \subseteq\{1, \ldots, n\}$, then the Koszulcomplex $K:=K\left(\left(x_{i}\right)_{i \in I} ; M\right)$ for a $R$-module $M$ is the $R$-complex whose underlying graded module is $\wedge\left(\oplus_{i \in I} R T_{1}\right) \otimes_{R} M$ and the basiselements $T_{i}$ map to $x_{i}$ for $i \in I$. If $J=\left\{j_{1}, \ldots, j_{s}\right\} \subseteq I$ with $j_{1}<\cdots<j_{s}$ the element $T_{J}$ denotes the product $T_{j_{1}} \cdots T_{j_{s}} \in K\left(\left(x_{i}\right)_{i \in I} ; R\right)$. For our convenience we will also use the index $J$ for the coefficient of the basiselement $T_{J} . Z_{k}(K), B_{k}(K)$ denote the submodule of cycles and boundaries of $K_{k}$ respectively. For the homology modules $H_{i}(K(\mathfrak{x} ; M))$ we will also write $H_{i}(\mathfrak{x} ; M)$ for short.

Main section.

LeMma 1. Let $(R, \mathrm{~m})$ be a local ring and $x_{1}, \ldots, x_{n}$ a sequence of elements of $R$ such that $\left(x_{1}, \ldots, \hat{x}_{i}, \ldots, x_{n}\right) \subseteq\left(x_{1}, \ldots, x_{n}\right)$ for all $1 \leqslant i \leqslant n$; then $Z_{k}(K(\mathfrak{x}, R)) \subseteq$ $\mathrm{m} K_{k}(\mathfrak{x}, R)$ for all $k \geqslant 1$.

Proof. Assume the assertion does not hold, so there is an $I \subseteq\{1, \ldots, n\}$ such that

$$
T_{I}-\sum_{J \neq I} \lambda_{J} T_{J} \in Z(K) \quad\left(\lambda_{J} \in R\right),
$$

i.e.

$$
\delta\left(T_{I}\right)-\sum_{J \neq I} \lambda_{J} \delta\left(T_{J}\right)=0
$$

hence

$$
\sum_{i \in I}(-1) \cdots x_{i} T_{I_{i}^{\wedge}}-\sum_{J \neq I} \lambda_{J} \sum_{j \in J}(-1) \cdots x_{j} T_{J_{j}^{\wedge}}=0
$$

So comparing coefficients of the same basiselements we obtain the inclusion of ideals $\left(x_{i}\right)_{i \in I} \subseteq\left(x_{j}\right)_{j \notin I}$, a contradiction to our assumption.

LEMMA 2. Let $(R, \mathrm{~m})$ be a local ring, $x_{1}, x_{2} \in R$ be an unconditioned weak sequence with $\left(x_{i}\right) \subseteq\left(x_{1}, x_{2}\right)$ for $i=1,2$, then the Koszulcomplex $K\left(x_{1}, x_{2} ; R\right)$ is a (module-) direct summand of the minimal $R$-free resolution of $R /\left(x_{1}, x_{2}\right)$. 
Proof. As $K_{0}\left(x_{1}, x_{2}\right)=R, K_{1}$ is the free module $K_{1}=R T_{1} \oplus R T_{2}$ with basiselements $T_{1}, T_{2}$ which map to $x_{1}, x_{2}$ respectively, we only have to show that $x_{2} T_{1}-x_{1} T_{2} \notin \mathrm{m} Z_{1}(K)$.

So assume $z=x_{2} T_{1}-x_{1} T_{2} \in m Z_{1}(K)$, then we have a representation $z=\sum \alpha_{i} z_{i}$, $\alpha_{i} \in \mathfrak{m}, z_{i} \in Z_{1}(K)$. We write $z_{i}=a_{i} T_{1}-b_{i} T_{2}, a_{i}, b_{i} \in R$, then $\sum \alpha_{i} a_{i}=x_{2}, \sum \alpha_{i} b_{i}$ $=x_{1}$. Since $z_{i} \in Z_{1}(K)$, we have $b_{i} \in\left(x_{1}: x_{2}\right)$ for all $i$ and therefore $\alpha_{i} b_{i}=\delta_{i} x_{1}$ for some $\delta_{i} \in R$, as $x_{1}, x_{2}$ form a weak sequence. Using $\sum \alpha_{i} b_{i}=x_{1}$, we obtain that there is an $i_{0}$ such that $\delta_{i_{0}} \notin \mathrm{m}$, hence a unit in $R$. Consider $w:=\delta_{i_{0}}^{-1} z_{i_{0}}=: a T_{1}-$ $b T_{2} \in Z_{1}(K)$, put $\alpha:=\alpha_{i_{0}}$, then

$$
\alpha w-\left(x_{2} T_{1}-x_{1} T_{2}\right)=\left(\alpha a-x_{2}\right) T_{1} \in \mathfrak{m} Z_{1}(K) .
$$

Now $w=a T_{1}-b T_{2}$ is a cycle, so $a \in\left(x_{2}: x_{1}\right)$, hence $\alpha a \in\left(x_{2}\right)$. So we may write $\alpha w-\left(x_{2} T_{1}-x_{1} T_{2}\right)=\mu x_{2} T_{1}$ with some $\mu \in R$. Therefore $\mu x_{1} x_{2}=0$, and by the weak sequence property we derive $\mu \mathrm{m}^{2}=0$.

If $x_{1} \notin \mathrm{m}^{2}$ or $x_{2} \notin \mathrm{m}^{2}$, then certainly $z=x_{2} T_{1}-x_{1} T_{2} \notin \mathrm{m}^{2} K \supseteq \mathrm{m} Z_{1}(K)$ a contradiction to our assumption. Hence we may assume that $x_{1}, x_{2} \in \mathrm{m}^{2}$, so $x_{2} \mu \in \mathrm{m}^{2} \mu=0$ and therefore $\alpha w-\left(x_{2} T_{1}-x_{1} T_{2}\right)=0$. So $\alpha a=x_{2}$ and $\alpha b=x_{1}$.

As $b \in\left(x_{1}: x_{2}\right)$, we have $m b \subseteq\left(x_{1}\right)$. Now consider the multiplication map $b$ : $\mathrm{m} \rightarrow\left(x_{1}\right)$ given by the multiplication with $b$. We have the exact sequence

$$
0 \rightarrow \operatorname{Ker}(b \cdot) \rightarrow \mathfrak{m} \stackrel{h}{\rightarrow}\left(x_{1}\right) \rightarrow 0 .
$$

Tensoring with $R / \mathrm{m}$, we have a surjective map $\mathrm{m} / \mathrm{m}^{2} \rightarrow\left(x_{1}\right) / \mathfrak{m}\left(x_{1}\right)$. Since $\alpha b=x_{1}$, $\alpha \in \mathfrak{m} \backslash \mathfrak{m}^{2}$. Let $m_{1}, \ldots, m_{n}$ denote a minimal system of generators of $m$ with $m_{1}=\alpha$ and $m_{i} b \in \mathrm{m}\left(x_{1}\right)$ for $i=2, \ldots, n$. Now write $m_{i} b=\gamma_{i} x_{1}$ with $\gamma_{i} \in \mathrm{m}$ for $i=2, \ldots, n$; then $\alpha \gamma_{i}-m_{i} \in \operatorname{Ker}(b \cdot)$, hence $\alpha \gamma_{i}-m_{i} \in\left(0: x_{1}\right)=(0: m)$ for $i=2, \ldots, n$.

So for all $i \geqslant 2, j \geqslant 1$ we have $m_{j}\left(\alpha \gamma_{i}-m_{i}\right)=0$, hence $m_{i} m_{j} \in m^{3} \cap(\alpha)$, therefore $\mathrm{m}^{2} / \mathrm{m}^{3}$ is generated by the class of $m_{1}^{2}=\alpha^{2}$, so $\mathrm{m}^{2}$ is generated by $\alpha^{2}$ by Nakayama's Lemma, hence $\mathrm{m}^{k}$ is generated by $\alpha^{k}$ for all $k \geqslant 2$. So we may write $x_{1}=u_{1} \alpha^{n_{1}}, x_{2}=u_{2} \alpha^{n_{2}}$ with $u_{1}, u_{2}$ units in $R$ and $n_{1}, n_{2}$ natural numbers. Since $n_{1} \leqslant n_{2}$ or $n_{2} \leqslant n_{1}$ we have $\left(x_{1}, x_{2}\right)=\left(x_{1}\right)$ or $\left(x_{1}, x_{2}\right)=\left(x_{2}\right)$ the desired contradiction.

Proposition 1. Let $(R, \mathrm{~m})$ be a local ring, $x_{1}, \ldots, x_{n}$ be an unconditioned weak sequence such that $\left(x_{1}, \ldots, \hat{x}_{i}, \ldots, x_{n}\right) \subset\left(x_{1}, \ldots, x_{n}\right)$ for all $1 \leqslant i \leqslant n$, then the elements $d\left(T_{I}\right)$ for $I \subseteq\{1, \ldots, n\}, \# I \geqslant 2$ form part of a basis of $Z(K) / \mathrm{m} Z(K)$, where $K=K(\mathfrak{x}, R)$.

Proof. Let $I \subseteq\{1, \ldots, n\}$ and denote

$$
z=\sum_{i \in I}(-1)^{\sigma(i, I)} x_{i} T_{i}
$$

where $\sigma(i, I)=\#\{j \in I / j<i\}$, then we have to show $z \notin m Z(K)$.

Let $R \rightarrow \bar{R}:=R /\left(x_{j}\right)_{j \notin I}$ be the canonical surjection, which induces a homomorphism $K(\mathfrak{x}, R) \rightarrow K(\mathfrak{x}, \bar{R})$. We reduce $K(\mathfrak{x}, \bar{R})$ modulo the ideal $\left\langle T_{j}\right\rangle_{j \notin l}$ and obtain a homomorphism of differential graded algebras

$$
K\left(x_{1}, \ldots, x_{n} ; R\right) \rightarrow K\left(\left(x_{i}\right)_{i \in I} ; R /\left(x_{j}\right)_{j \notin I}\right) .
$$


Now it is enough to show that the image of $z$ under this homomorphism is not contained in $\overline{\mathrm{m}} K\left(\left(x_{i}\right)_{i \in I} ; R /\left(x_{j}\right)_{j \notin I}\right)$, where $\overline{\mathrm{m}}$ denotes the maximal ideal of $R /\left(x_{j}\right)_{j \notin I}$.

So without loss of generality by renumbering we only have to show

$$
z=\sum_{i}(-1)^{i+1} x_{i} T_{1} \cdot \ldots \cdot \hat{T}_{i} \cdot \ldots \cdot T_{n} \notin \mathrm{m} Z_{n-1}(K) .
$$

Let us assume this is not true and reduce again modulo $x_{3}, \ldots, x_{n}$, then

$$
\tilde{z}=\bar{x}_{1} T_{2} \cdot \ldots \cdot T_{n}-\bar{x}_{2} T_{1} T_{3} \cdot \ldots \cdot T_{n} \in \overline{\mathrm{m}} Z_{n-1}\left(K\left(\mathfrak{x}, R /\left(x_{3}, \ldots, x_{n}\right)\right)\right),
$$

hence there exist $\alpha_{j} \in \mathrm{m}, z_{j} \in Z_{n-1}\left(K\left(\mathfrak{x}, R /\left(x_{3}, \ldots, x_{n}\right)\right)\right), \quad z_{j}=\sum_{i}(-1)^{i+1} a_{j i} T_{1}$ $\cdot \ldots \cdot \hat{T}_{i} \cdot \ldots \cdot T_{n}$, such that $z=\sum_{j} \alpha_{j} z_{j}$. Since $\delta z_{j}=0$, we have $a_{j 1} x_{2} T_{3} \cdot \ldots \cdot T_{n}-$ $a_{j 2} x_{1} T_{3} \cdot \ldots \cdot T_{n}=0$, hence $a_{j 1} x_{2}-a_{j 2} x_{1}=0$ for all $j$ and $\Sigma_{j} \alpha_{j} a_{j 1}=\bar{x}_{1}, \Sigma_{j} \alpha_{j} a_{j 2}=$ $\bar{x}_{2}$. So consider $w_{j}:=a_{j 1} T_{1}-a_{j 2} T_{2} \in Z_{1}\left(K\left(\bar{x}_{1}, \bar{x}_{2} ; R /\left(x_{3}, \ldots, x_{n}\right)\right)\right)$, then

$$
\sum_{j} \alpha_{j} w_{j}=\bar{x}_{2} T_{1}-\bar{x}_{1} T_{2} \in \mathrm{m} Z\left(K\left(\bar{x}_{1}, \bar{x}_{2} ; R /\left(x_{3}, \ldots, x_{n}\right)\right)\right) \text {, }
$$

which contradicts Lemma 2. This proves the assertion of the Proposition.

REMARK. There exist unconditioned weak sequences $\mathfrak{x}$, for which the assumption $\left(x_{1}, \ldots, \hat{x}_{i}, \ldots, x_{n}\right) \subsetneq\left(x_{1}, \ldots, x_{n}\right)$ for all $1 \leqslant i \leqslant n$ does not hold and hence the assertion of the proposition cannot be true in these cases.

EXAmple. Let $(R, \mathfrak{m})$ be a regular local ring of dimension 1 and let $x$ be a generator of $\mathrm{m}$. Consider $x_{1}:=x, x_{2}:=x^{r}(r \geqslant 1)$. Then $\left(0: x_{1}\right)=\left(0: x_{2}\right)=0$, $\left(x_{1}: x_{2}\right)=R$, hence $\mathrm{m}\left(x_{1}: x_{2}\right)=\left(x_{1}\right),\left(x_{2}: x_{1}\right)=\left(x^{r-1}\right)$, hence $\mathrm{m}\left(x_{2}: x_{1}\right)=\left(x_{2}\right)$.

LEMMA 3. Let $(R, \mathrm{~m})$ be a local ring, $x_{1}, \ldots, x_{n}$ an unconditioned weak sequence such that $\left(x_{1}, \ldots, \hat{x}_{i}, \ldots, x_{n}\right) \subset\left(x_{1}, \ldots, x_{n}\right)$ for all $1 \leqslant i \leqslant n$. Let $I \subseteq\{1, \ldots, n\}$ and denote $z=\sum_{i \in I} \alpha_{i} T_{i} \in K_{1}(\mathfrak{x}, T)$ a cycle, then for every $i \in I$ and for every $\beta \in \mathrm{m}$ we have $\beta \alpha_{i} \in \mathfrak{m}\left(x_{j}\right)_{j \in I_{i}^{\wedge}}$.

Proof. By a suitable renumbering we may assume $I=\{1, \ldots, s\}$. Modulo the ideal $\left(x_{3}, \ldots, x_{n}\right)$ we consider $\bar{z}=\bar{\alpha}_{1} T_{1}+\bar{\alpha}_{2} T_{2} . \bar{z}$ is a cycle in $K_{1}\left(\mathfrak{x}, R /\left(x_{3}, \ldots, x_{n}\right)\right)$. Now it is enough to show that $\beta \alpha_{1} \in \mathrm{m}\left(x_{2}\right)$ and $\beta \alpha_{2} \in \mathrm{m}\left(x_{1}\right)$ modulo $\left(x_{3}, \ldots, x_{n}\right)$ for every $\beta \in \mathrm{m}$. So without loss of generality $n=2, z=a T_{1}-b T_{2}$ and we show $\beta a \in \mathrm{m}\left(x_{2}\right), \beta b \in \mathrm{m}\left(x_{1}\right)$ for $\beta \in \mathrm{m}$.

So take any $\beta \in m$, then there exist $u_{1}, u_{2} \in R$ such that $\beta a=u_{2} x_{2}, \beta b=u_{1} x_{1}$ by the weak sequence property.

Now $u_{1}, u_{2}$ cannot simultaneously be units in $R$. If so, we have $u_{1} x_{1} x_{2}=u_{2} x_{1} x_{2}$, since $z=a T_{1}-b T_{2}$ is a cycle, and hence by the weak sequence property $\left(u_{1}-u_{2}\right) \mathrm{m}^{2}=0$. So $\alpha=u_{2}^{-1} u_{1} \alpha$ for every $\alpha \in \mathrm{m}^{2}$. Now since $x_{1}, x_{2}$ is an unconditioned weak sequence, so is $x_{1}^{\prime}, x_{2}^{\prime}$, where we denote $x_{1}^{\prime}:=u_{1} x_{1}, x_{2}^{\prime}:=u_{2} x_{2}$. Consider the cycle $z^{\prime}:=u_{2} a T_{1}^{\prime}-u_{1} b T_{2}^{\prime} \in K\left(x_{1}^{\prime}, x_{2}^{\prime} ; R\right)$. Then

$$
\begin{aligned}
u_{2}^{-1} \beta z^{\prime} & =\beta a T_{1}^{\prime}-u_{2}^{-1} u_{1} \beta b T_{2}^{\prime}=\beta a T_{1}^{\prime}-\beta b T_{2}^{\prime} \\
& =x_{2}^{\prime} T_{1}^{\prime}-x_{1}^{\prime} T_{2}^{\prime} \in \operatorname{m} Z(K),
\end{aligned}
$$

which contradicts the assertion of Lemma 2.

So at least one of the coefficients $u_{1}, u_{2}$ is a nonunit. Let $u_{1} \in \mathrm{m}$. We have to show that also $u_{2} \in \mathrm{m}$. So assume $u_{2}$ is a unit, without loss of generality $u_{2}=1$. Then 
$x_{1} x_{2}=\beta a x_{1}=\beta b x_{2}=u_{1} x_{1} x_{2}$, hence $x_{1} x_{2}=0$. We obtain $x_{2} \in\left(0: x_{1}\right)=(0: m)$, therefore $x_{2} \mathrm{~m}=0$ hence $\left(0: x_{2}\right)=\mathrm{m}$. But then we have $x_{2}=\beta a \in \mathrm{m}^{2}=\mathrm{m}\left(0: x_{2}\right)$ $=m(0: m)=0$ a contradiction.

COROLlaRY. Let $(R, \mathrm{~m})$ be a local ring, $x_{1}, \ldots, x_{n}$ an unconditioned weak sequence such that $\left(x_{1}, \ldots, \hat{x}_{i}, \ldots, x_{n}\right) \subsetneq\left(x_{1}, \ldots, x_{n}\right)$ for all $1 \leqslant i \leqslant n$, then $\mathrm{m}\left(\left(x_{j}\right)_{\left.j \in I_{i}: x_{i}\right) \subseteq}\right.$ $\mathrm{m}\left(x_{j}\right)_{j \in l_{i}}$.

Proof. Let $\alpha \in\left(\left(x_{j}\right)_{j \in I_{i}}: x_{i}\right), \beta \in \mathrm{m}$, then there exist $\gamma_{j} \in R$ for $j \in I_{i}$ such that $\alpha x_{i}=\sum_{j \in I_{i}} \gamma_{j} x_{j}$. Then $\alpha T_{i}-\sum_{j \in l_{i}} \gamma_{j} T_{j}$ is a cycle in $K(x, R)$, hence by Lemma 3 we have $\beta \alpha \in \mathrm{m}\left(x_{j}\right)_{j \in I_{i} \text {. }}$.

We are now able to prove the following

THEOREM. Let $(R, \mathrm{~m})$ be a local ring, $x_{1}, \ldots, x_{n}$ be a sequence of elements such that $\left(x_{1}, \ldots, \hat{x}_{i}, \ldots, x_{n}\right) \subsetneq\left(x_{1}, \ldots, x_{n}\right)$ for all $1 \leqslant i \leqslant n$, then the following are equivalent:

(1) $x_{1}, \ldots, x_{n}$ is an unconditioned weak sequence,

(2) for all $I \subseteq\{1, \ldots, n\}, 1 \leqslant k \leqslant \# I, J_{0} \subseteq I$ with $\# J_{0}=\# I-k$ :

$$
H_{k}\left(K\left(x_{i}\right)_{i \in I}\right) / \imath\left(\left(\mathrm{m}(\mathfrak{c}) \sum_{\substack{\# J=k \\ J \cap J_{0} \neq \varnothing}} K_{k}\left(x_{j}\right)_{j \in J}\right) \cap Z_{k}\left(K\left(x_{i}\right)_{i \in I}\right)\right)
$$

is a vectorspace,

(3) for all $I \subseteq\{1, \ldots, n\}, i_{0} \in I$ :

$$
H_{1}\left(K\left(x_{i}\right)_{i \in I}\right) / \imath\left(\left(\mathrm{m}(\mathfrak{x}) K_{1}\left(x_{i}\right)_{i \in I_{i 0}}\right) \cap Z\left(K\left(x_{i}\right)_{i \in I}\right)\right)
$$

is a vectorspace,

(4) for all $I \subseteq\{1, \ldots, n\}, i_{0} \in I$ :

$$
H_{1}\left(K\left(x_{i}\right)_{i \in I}\right) / H_{1}\left(K\left(x_{i}\right)_{i \in I_{i_{0}}}\right)
$$

is a vectorspace.

( $\iota$ denotes the canonical map $Z_{*}(K) \rightarrow H_{*}(K)$.)

Proof. (2) $\Rightarrow(3) \Rightarrow(4)$ are obvious.

(4) $\Rightarrow(1)$. Consider the long exact homology sequence associated to

$$
0 \rightarrow K\left(x_{i}\right)_{i \in I_{i_{0}}} \rightarrow K\left(x_{i}\right)_{i \in I} \rightarrow K\left(x_{i}\right)_{i \in I_{i_{0}}}[-1] \rightarrow 0,
$$

then we obtain that the vectorspace $H_{1}\left(K\left(x_{i}\right)_{i \in I}\right) / H_{1}\left(K\left(x_{i}\right)_{i \in I_{i 0}}\right)$ is just the kernel of the multiplication map

$$
R /\left(x_{i}\right)_{i \in I_{i_{0}}} \stackrel{x_{i_{0}}}{\rightarrow} R /\left(x_{i}\right)_{i \in I_{i_{0}}}
$$

hence $m\left(\left(x_{i}\right)_{i \in I_{i 0}}: x_{i}\right) \subseteq\left(x_{i}\right)_{i \in I_{i 0}^{0}}$.

(1) $\Rightarrow$ (2). Let $\# I=r, 1 \leqslant k \stackrel{10}{\leqslant}$ and $J_{0} \subseteq I$ with $\# J_{0}=r-k$. Consider

$$
z=\sum_{\substack{\# \Re=k \\ \Omega \subseteq I}} \alpha_{\Omega} T_{\Re} \in Z_{k}\left(K\left(x_{i}\right)_{i \in I}\right) .
$$


Denote $\Re_{0}:=I \backslash J_{0}$, then $\alpha_{\mathfrak{s}_{0}} \in\left(\left(x_{j}\right)_{j \notin \Omega_{0}}:\left(x_{j^{\prime}}\right)_{j^{\prime} \in \aleph_{0}}\right)$, so for any $\beta \in \mathbb{m}: \beta \alpha_{\mathfrak{s}_{0}} \in$ $m\left(x_{j}\right)_{j \notin k_{0}}$ by the corollary of Lemma 3. So write

$$
\beta \alpha_{\mathrm{s}_{0}}=\sum_{j \notin \mathrm{s}_{0}} \gamma_{j} x_{j} \quad \text { with } \gamma_{j} \in \mathrm{m}
$$

hence

$$
\beta z-\delta\left(\sum_{, \in \aleph_{0}} \gamma_{j} T_{j} T_{\mathfrak{s}_{0}}\right) \in\left(\mathrm{m}(\mathfrak{x}) \sum_{\substack{\# J=k \\ J \cap J_{0} \neq \varnothing}} K_{k}\left(x_{j}\right)_{j \in J}\right) \cap Z_{k}\left(K\left(x_{i}\right)_{1 \in I}\right),
$$

since for each $₫$ we also have $\beta \alpha_{\mathfrak{B}} \in \mathrm{m}(\mathrm{x})$ again by the corollary of Lemma 3 .

REMARK 1. If $x_{1}, \ldots, x_{n}$ is an unconditioned relatively $m$ regular sequence with respect to $m(x)$ in the sense of Fiorentini [1], then $m(x) K_{k}\left(x_{i}\right)_{i \in I} \cap Z_{k}\left(K\left(x_{i}\right)_{i \in I}\right)$ $\subseteq \mathrm{m} B_{k}\left(K\left(x_{i}\right)_{i \in I}\right)$ for all $I \subseteq\{1, \ldots, n\}$ by Fiorentini's result, hence the submodules by which we have to divide $H_{k}\left(K\left(x_{i}\right)_{i \in I}\right)$ in (2) and (3) in the theorem vanish.

For a system of parameters $x$ of a Buchsbaum ring this assumption certainly holds, since in this case $r$ is even a $d$-sequence (cf. [2]).

REMARK 2. In general for unconditioned weak sequences the homology modules are not vectorspaces.

Example. Let $A=k\left[\left[X_{1}, X_{2}, Y_{1}, Y_{2}, Z\right]\right]$ be the powerseries ring in five variables over a field $k$ and denote $m$ the maximal ideal of $A$.

Let

$$
R:=A /\left(Y_{1}^{2}, Y_{1} Y_{2}, Y_{2}^{2}, Y_{1} X_{2}-Y_{2} X_{1}, Y_{1} m^{2}, Y_{2} m^{2}, Z^{2}, Z m^{2}, Z Y_{1}, Z Y_{2}-Y_{1} X_{2}\right) \text {. }
$$

denote $m$ the maximal ideal of $R$ and $x_{1}, x_{2}, y_{1}, y_{2}, z$ the residues of the corresponding elements of $A$. Then

(a) $x_{1}, x_{2}$ is an unconditioned weak sequence.

(b) $H_{1}\left(x_{1}, x_{2} ; R\right)$ is not a vectorspace.

Proof. (a) $\left(0: x_{1}\right)=\left(0: x_{2}\right)=\left(y_{1}, y_{2}, z\right) \mathrm{m}$,

$$
\begin{aligned}
& \left(x_{1}: x_{2}\right)=\left(y_{1}, y_{2}, z\right) \mathrm{m}+\left(y_{1}, x_{1}\right), \\
& \left(x_{2}: x_{1}\right)=\left(y_{1}, y_{2}, z\right) \mathrm{m}+\left(y_{2}, x_{2}\right) .
\end{aligned}
$$

Obviously $x_{1}, x_{2}$ is an unconditioned weak sequence.

(b) Consider $y_{2} T_{1}-y_{1} T_{2} \in Z\left(K\left(x_{1}, x_{2} ; R\right)\right)$. Then $0 \neq z y_{2} T_{1}-z y_{1} T_{2}=z y_{2} T_{1} \in$ $\mathrm{m} Z(K)$. Notice that $R$ is a homogeneous ring. Assume now that $z y_{2} T_{1} \in B(K)$, then there exists a linear form $f$ such that $\delta\left(f T_{1} T_{2}\right)=z y_{2} T_{1}$, but $\delta\left(f T_{1} T_{2}\right)=f x_{1} T_{2}-f x_{2} T_{1}$, hence $f \in\left(0: x_{1}\right) \subseteq \mathrm{m}^{2}$ a contradiction.

Remark 3. The example in the remark to Proposition 1 shows for $r \geqslant 3$ that the theorem is not valid without the assumption $\left(x_{1}, \ldots, \hat{x}_{1}, \ldots, x_{n}\right) \subset\left(x_{1}, \ldots, x_{n}\right)$ for all $1 \leqslant i \leqslant n$. In this particular case $H_{1}\left(K\left(x_{1}\right)\right)=0$ and $x^{r-1} H_{1}\left(K\left(x_{1}, x_{2}\right)\right) \neq 0$.

Using the theorem and the following Proposition 2 we now would like to give a new proof of the characterization of Buchsbaum rings in terms of the Koszulhomology. This characterization is already known using Suzuki [7] and Shimoda [3]:

The following are equivalent:

(1) $R$ is a Buchsbaum ring,

(2) for all systems of parameters $\mathfrak{r}: H_{*}(\mathfrak{x}, R)$ is a vectorspace, 
(3) for all systems of parameters $\mathfrak{x}: H_{1}(\mathfrak{x}, R)$ is a vectorspace,

(4) for all systems of parameters $\mathfrak{x}: \tilde{H}_{*}(\mathfrak{x}, R)$ is a vectorspace,

(5) for all systems of parameters $\mathfrak{x}: \tilde{H}_{1}(\mathfrak{x}, R)$ is a vectorspace.

$\left(\tilde{H}_{i}(\mathfrak{x}, M):=\operatorname{coker}\left(H_{i}(\mathfrak{x},(\mathfrak{x}) M) \rightarrow H_{i}(\mathfrak{x}, M)\right)\right.$ for a $R$-module $M$.)

(1) $\Rightarrow$ (2) follows from our theorem and Remark 1 . Obviously (2) $\Rightarrow(3) \Rightarrow(5)$ and $(2) \Rightarrow(4) \Rightarrow(5)$. We only need to show $(5) \Rightarrow(1)$, hence e.g. every system of parameters $\mathfrak{x}$ is a weak sequence. The following proposition which we prove more general for the module case (cf. Introduction) implies the desired result.

Proposition 2. Let $(R, \mathrm{~m})$ be a local ring, $M$ a finitely generated $R$-module and $x_{1}, \ldots, x_{n}$ a sequence of elements in $\mathrm{m}$ such that $\tilde{H}_{1}\left(x_{1}^{\nu_{1}}, \ldots, x_{n}^{\nu_{n}}, M\right)$ is a vectorspace for all multi-indices $\left(\nu_{1}, \ldots, \nu_{n}\right)$ with $\nu_{i} \geqslant 1$, then $x_{1}^{\nu_{1}}, \ldots, x_{n}^{\nu_{n}}$ is a weak sequence on $M$ for all $\left(\nu_{1}, \ldots, \nu_{n}\right)$.

Proof. It is only left to show that $x$ is a weak sequence. We will do this in two steps: (1) $\mathfrak{m}\left(\left(x_{1}, \ldots, x_{n-1}\right) M: x_{n}\right) \subseteq\left(x_{1}, \ldots, x_{n-1}\right) M$. (2) If $\tilde{H}_{1}\left(x_{1}^{\nu_{1}}, \ldots, x_{n}^{\nu_{n}} ; M\right)$ is a vectorspace for all $\nu_{1}, \ldots, \nu_{n}$, then $\tilde{H}_{1}\left(x_{1}^{\nu_{1}}, \ldots, x_{n-1}^{\nu_{n-1}} ; M\right)$ is a vectorspace for all $\nu_{1}, \ldots, \nu_{n-1}$.

Then descending step by step we obtain the conclusion.

To (1). We have the following commutative diagram with exact rows:

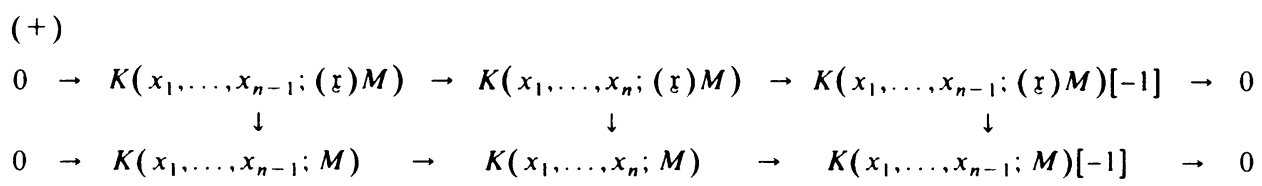

Taking homology we get the following commutative diagram:

$$
\begin{array}{ccccccc}
H_{1}\left(x_{1}, \ldots, x_{n} ;(\mathfrak{x}) M\right) & \stackrel{\varphi_{\mathrm{r}}}{\rightarrow} & H_{0}\left(x_{1}, \ldots, x_{n-1} ;(\mathfrak{x}) M\right) & \rightarrow & \operatorname{coker} \varphi_{\mathfrak{x}} & \rightarrow & 0 \\
\downarrow & & \downarrow & & \downarrow \psi_{0} & & \\
H_{1}\left(x_{1}, \ldots, x_{n} ; M\right) & \stackrel{\varphi}{\rightarrow} & H_{0}\left(x_{1}, \ldots, x_{n-1} ; M\right) & \rightarrow & \operatorname{coker} \varphi & \rightarrow & 0 \\
\downarrow & & \downarrow & & \downarrow & & \\
\tilde{H}_{1}\left(x_{1}, \ldots, x_{n} ; M\right) & \rightarrow & \operatorname{coker} \psi & & \rightarrow & \operatorname{coker} \psi_{0} &
\end{array}
$$

In other terms

$$
\begin{aligned}
& H_{1}(\mathrm{r}:(\mathrm{r}) M) \rightarrow(\mathrm{r}) M /(\mathfrak{x})\left(x_{1}, \ldots, x_{n-1}\right) M \stackrel{x_{n} \cdot}{\rightarrow}(\mathfrak{r})^{2} M+(\mathfrak{r})\left(x_{1} \ldots \ldots x_{n-1}\right) M \quad \rightarrow \quad 0 \\
& \begin{array}{ccccc}
\downarrow & & \downarrow & & \downarrow \\
H_{1}(\mathrm{r}, M) & \rightarrow & M /\left(x_{1} \ldots \ldots x_{n-1}\right) M & \stackrel{x_{n}}{\rightarrow} & (\mathrm{r}) M /\left(x_{1}, \ldots, x_{n-1}\right) M \\
\downarrow & & \downarrow & & \\
\dot{H}_{1}(\mathrm{r}: M) & \rightarrow & M /(\mathrm{r}) M & \stackrel{x_{n}}{\rightarrow} & (\mathrm{r}) M /\left(x_{1}, \ldots, x_{n-1}, x_{n}^{2}\right) M
\end{array}
\end{aligned}
$$

By the snake lemma $\tilde{H}_{1}(\mathfrak{x}, M)$ maps onto the kernel of

$$
M /(\mathfrak{x}) M^{x_{n} \cdot} \rightarrow(\mathfrak{x}) M /\left(x_{1}, \ldots, x_{n-1}, x_{n}^{2}\right) M,
$$


hence

$$
\mathrm{m}\left(\left(x_{1}, \ldots, x_{n-1}, x_{n}^{2}\right) M: x_{n}\right) \subseteq\left(x_{1}, \ldots, x_{n}\right) M .
$$

Now let $\alpha \in\left(x_{1}, \ldots, x_{n-1}\right) M: x_{n}$ we have to show that $\alpha \mathrm{m} \subseteq\left(x_{1}, \ldots, x_{n-1}\right) M$. But

$$
\alpha x_{n} \in\left(x_{1}, \ldots, x_{n-1}\right) M \subseteq\left(x_{1}, \ldots, x_{n-1}, x_{n}^{k+1}\right) M \text { for all } k \geqslant 1 \text {, }
$$

so $\alpha x_{n}^{k} \in\left(x_{1}, \ldots, x_{n-1}, x_{n}^{2 k}\right) M$. Now repeating the above argument for $m \alpha \subseteq$ $\left(x_{1}, \ldots, x_{n-1}, x_{n}^{k}\right) M$ for all $k \geqslant 1$, hence $\operatorname{m} \alpha \subseteq\left(x_{1}, \ldots, x_{n-1}\right) M$.

To (2). Again taking homology from (+) we derive Diagram A.

By the snake lemma we have an exact sequence

$$
K \rightarrow C\left(x_{1}, \ldots, x_{n} ; M\right) \rightarrow \tilde{H}_{1}(\mathfrak{x} ; M) .
$$

The map $K \rightarrow C\left(x_{1}, \ldots, x_{n} ; M\right)$ is actually the zero map. To prove this, consider $\alpha \in M$ with $\bar{\alpha} \in K$, then $\alpha \in\left(x_{1}, \ldots, x_{n-1}\right) M$. Let $\alpha=\sum_{i=1}^{n-1} x_{i} \beta_{i}, \beta_{i} \in M$ then

$$
x_{n} \alpha=\sum_{i=1}^{n-1} x_{n} x_{i} \beta_{i},
$$

so $z=\alpha T_{n}-\sum_{i=1}^{n-1} \beta_{i} x_{n} T_{i}$ is a cycle in $K(\mathfrak{x},(\mathfrak{x}) M)$ which maps onto $\bar{\alpha}$. But $z=$ $\delta\left(\sum_{i=1}^{n-1} \beta_{i} T_{i} T_{n}\right)$ is a boundary in $K_{1}\left(x_{1}, \ldots, x_{n} ; M\right)$. So $C\left(x_{1}, \ldots, x_{n} ; M\right)$ is a vectorspace since $\tilde{H}_{1}(\mathfrak{x} ; M)$ is.

Again the same argument is true for the sequence $x_{1}, \ldots, x_{n-1}, x_{n}^{k}$ for all $k \geqslant 1$, hence for all $k \geqslant 1: C\left(x_{1}, \ldots, x_{n-1}, x_{n}^{k}, M\right)$ is a vectorspace. Therefore also

$$
\begin{aligned}
& C^{\prime}\left(x_{1}, \ldots, x_{n-1}, x_{n}^{k} ; M\right) \\
& \quad:=\operatorname{coker}\left(H_{1}\left(x_{1}, \ldots, x_{n-1} ;\left(x_{1}, \ldots, x_{n-1}, x_{n}^{k}\right) M\right) \rightarrow H_{1}\left(x_{1}, \ldots, x_{n-1} ; M\right)\right)
\end{aligned}
$$

is a vectorspace.

Finally consider Diagram B.

By the snake lemma we have the exact sequence

$$
L \rightarrow \tilde{H}_{1}\left(x_{1}, \ldots, x_{n-1} ; M\right) \rightarrow C^{\prime}\left(x_{1}, \ldots, x_{n-1}, x_{n}^{k} ; M\right) .
$$

So $\tilde{H}_{1}\left(x_{1}, \ldots, x_{n-1} ; M\right)$ modulo the submodule generated by all cycles $z=\sum_{i=1}^{n-1} \alpha_{i} T_{i}$ with $\alpha_{i} \in\left(x_{1}, \ldots, x_{n-1}, x_{n}^{k}\right) M$ is a vectorspace, hence for every cycle $z \in K_{1}$ we have

$$
\mathrm{m} z \subseteq \bigcap_{k \geqslant 1}\left(x_{1}, \ldots, x_{n-1}, x_{n}^{k}\right) K_{1}=\left(x_{1}, \ldots, x_{n-1}\right) K_{1},
$$

so $m \tilde{H}_{1}\left(x_{1}, \ldots, x_{n-1} ; M\right)=0$.

We now want to look at the minimal $R$-free resolution of $R /(\mathfrak{x})$ for an unconditioned weak sequence $x$.

By Lemma 2 the Koszulcomplex is always a (module-)direct summand of this resolution for $n \leqslant 2$. But beside this fact in general very little can be said about what this resolution looks like. If $\mathfrak{x}$ is just a sequence of one element $x_{1}$, then the resolution of $R /\left(x_{1}\right)$ is given by

$$
\cdots \rightarrow F_{2} \rightarrow F_{1} \rightarrow F_{0} \stackrel{\varphi}{\rightarrow} R \stackrel{x_{1}}{\rightarrow} R \rightarrow R /\left(x_{1}\right) \rightarrow 0,
$$

where $\left(F_{*}, d_{*}\right)$ is a direct sum of $\operatorname{dim}_{R / \mathrm{m}}(0: \mathrm{m})=\operatorname{dim}_{R / \mathrm{m}} H_{1}\left(x_{1}\right)$ copies of the minimal $R$-resolution of the residue class field $R / \mathrm{m}$ and $\varphi$ maps the basiselements of $F_{0}$ to representatives of a minimal system of generators of $H_{1}\left(x_{1}\right)$. So in this case finding the $R$-resolution of $R /\left(x_{1}\right)$ is equivalent to finding the $R$-resolution of $R / \mathrm{m}$. 


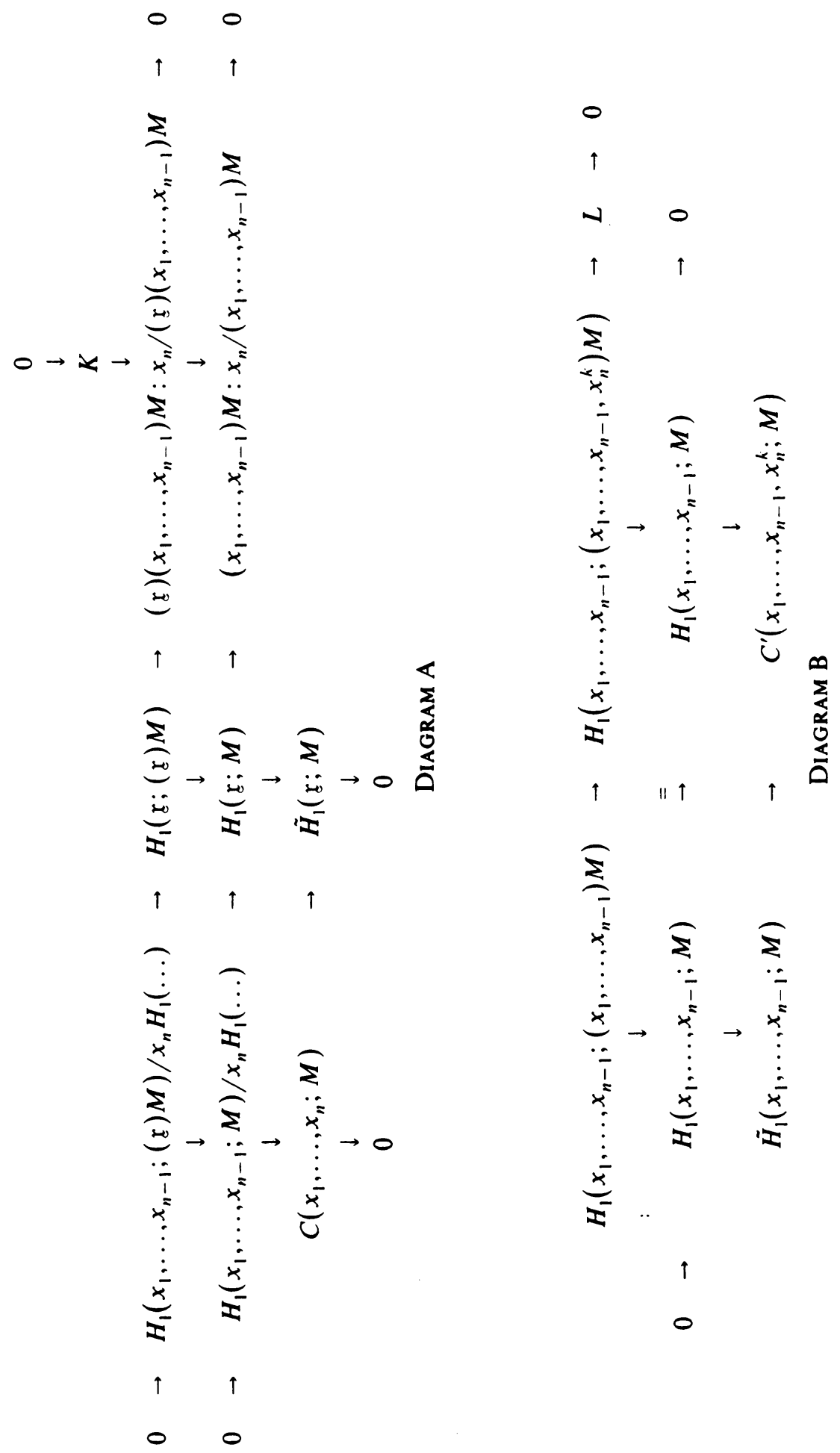


So one may ask in general: Assume we know the $R$-resolutions of the homology modules $H_{i}(x)$, what can be said about the $R$-resolution of $R /(x)$ in terms of these given resolutions?

We want to give an answer to this question in a very special case: namely $\mathrm{r}=x_{1}, x_{2}$ is an unconditioned weak sequence of two elements and $\mathrm{r}$ is unconditioned $m$-relatively regular with respect to $m(x)$.

In this case $H_{1}(x), H_{2}(x)$ are vectorspaces by Remark 1 to the theorem. Denote $M, N$ free $R$-modules of rank $\operatorname{dim} H_{1}(x)$ and $\operatorname{dim} H_{2}(x)$ respectively and denote $(\mathbf{G}, d)$ the resolution of the residue class field $R / \mathrm{m}$. Below we will give an equivalent condition for the $R$-resolution of $R /(\mathfrak{x})$ to be isomorphic to

$$
K \oplus(G \otimes M[-2]) \oplus(G \otimes N[-3])
$$

with obvious maps $G_{0} \otimes M[-2] \rightarrow K_{1}, G_{0} \otimes N[-3] \rightarrow K_{2}$ killing the homology $H_{*}(K)$.

Denote $\left\{z_{j} \mid j=1, \ldots, s\right\}$ a set of cycles in $K_{1}(\mathfrak{x})$ which represent a $R / \mathrm{m}$ vectorspace basis of $H_{1}(x)$. Let $m_{1}, \ldots, m_{n}$ be a minimal system of generators of the maximal ideal $m$. For every pair $m_{i}, z_{j}$ we choose an element $\gamma\left(m_{i}, z_{j}\right) \in m$ such that $\delta\left(\gamma\left(m_{i}, z_{j}\right) T_{1} T_{2}\right)=m_{i} z_{j}$. This is possible, see Remark 1. Let $\mathfrak{A}$ be the $R$-ideal

$$
\mathfrak{U}:=\left(\sum_{i} r_{i} \gamma\left(m_{i}, z_{j}\right) / j=1, \ldots, s ; \sum_{i} r_{i} m_{i}=0\right) .
$$

Notice that the choices for $\gamma\left(m_{i}, z_{j}\right)$ are determined up to socle-elements, so as any relation $\sum r_{i} m_{i}=0$ only involves $r_{i} \in \mathfrak{m}$ the ideal $\mathfrak{A}$ is well defined.

If $\sum r_{i} m_{i}=0$ then

$$
\sum_{i} r_{i} \gamma\left(m_{i}, z_{j}\right)\left(x_{1} T_{2}-x_{2} T_{1}\right)=\delta\left(\sum_{i} r_{i} \gamma\left(m_{i} z_{j}\right) T_{1} T_{2}\right)=\left(\sum_{i} r_{i} m_{i}\right) z_{j}=0 \text {. }
$$

Hence $\Sigma_{i} r_{i} \gamma\left(m_{i}, z_{j}\right) \in(0: \mathfrak{m})$, so we obtain $\mathfrak{A} \subseteq(0: \mathfrak{m})$.

Claim. The minimal $R$-resolution $(F, d)$ of $R /(\mathfrak{x})$ is isomorphic to $(X)$ if and only if $\mathfrak{A}=0$.

Proof. Clearly the $R$-resolution $F$ of $R /(x)$ starts with

$F_{0}=R$,

$F_{1}=R T_{1} \oplus R T_{2} ; T_{1} \mapsto x_{1}, T_{2} \mapsto x_{2}$,

$F_{2}=R T_{1} T_{2} \oplus G_{0} \otimes M[2]$.

Denote $S_{j}, j=1, \ldots, s$, a free basis of $M[-2]$ and let $1 \otimes S$, map to $z$, for $j=1, \ldots, s$.

So we may define for $i=1, \ldots, n, j=1, \ldots, s$ :

$$
d\left(V_{i} \otimes S_{j}\right)=m_{i}\left(1 \otimes S_{j}\right)-\gamma\left(m_{i}, z_{j}\right) T_{1} T_{2},
$$

where $V_{1}, \ldots, V_{n}$ denote a $R$-basis of $G_{1}$ which is mapped to $m_{1}, \ldots, m_{n}$ in $\mathbf{G}$.

If $\mathfrak{A}=0$, then we can define

$$
\begin{aligned}
& \left.d\right|_{(G \otimes M[-2])_{i>4}}=\left.\left(d_{G}\right)_{i \geqslant 2} \otimes \mathrm{id}\right|_{M[-2]}, \\
& \left.d\right|_{(G \otimes N[-3])_{i>4}}=\left.\left(d_{G}\right)_{i \geqslant 1} \otimes \mathrm{id}\right|_{N[-3]}
\end{aligned}
$$

and $G_{0} \otimes N[-3] \rightarrow K_{2}$ the obvious map killing the homology $H_{2}(K)$. So we have the resolution as desired. 
If $\mathfrak{U} \neq 0$, then there is a relation $\Sigma r_{i} m_{i}=0, j \in\{1, \ldots, s\}$ with $0 \neq \sigma_{j}=$ $\Sigma_{i} r_{i} \gamma\left(m_{i}, z_{j}\right) \in(0: \mathfrak{m})$. Hence the element $\sigma_{j} T_{1} T_{2}$ is already killed by $d\left(G_{1} \otimes M[-2]\right)$ and therefore must not be killed by a basiselement of $G_{0} \otimes N[-3]$. Also $\left(\Sigma_{i} r_{i} m_{i}\right)$. $\left(1 \otimes S_{j}\right)=0$, but $\left(\sum_{i} r_{i} V_{i}\right) \otimes S_{j}$ is not a cycle in $F$ although $\sum r_{i} V_{i}$ is a cycle in $G$. So in this case $(\times)$ even is not a complex. Hence the $R$-resolution of $R /(\mathfrak{x})$ is different from $(\times)$.

\section{REFERENCES}

1. M. Fiorentini, On relative regular sequences, J. Algebra 18 (1971), 384-389.

2. C. Huneke, The theory of $d$-sequences and powers of ideals, Adv. in Math. (to appear).

3. Y. Shimoda, On the syzygy part of Koszul homology on certain ideals (preprint).

4. J. Stückrad and W. Vogel, Eine Verallgemeinerung der Cohen-Macaulay Ringe und Anwendungen auf ein Problem der Multiplizitätstheorie, J. Math. Kyoto Univ. 13 (1973), 513-528.

5. __ Über das Amsterdamer Programm von W. Gröbner und Buchsbaum Varietäten, Monatsh. Math. 78 (1974), 433-445.

6. Toward a theory of Buchsbaum singularities, Amer. J. Math. 100 (1978), 727-746.

7. N. Suzuki, On the Koszul complex generated by a system of parameters for a Buchsbaum module, Sci. Rep. Shizuoka College of Pharmacy 8 (1979), 27-35.

8. W. Vogel, Über eine Vermutung von D. A. Buchshaum, J. Algebra 25 (1973), 106-112.

Department of Mathematics, Universitat Essen, Essen, West Germany

Department of Mathematics, Brandeis University, Waltham, Massachusetts 02154 